\title{
Antipodal Vivaldi Antenna with Ceramic Cone Lens for Biomedical Microwave Imaging Systems
}

\author{
Mengchu Wang ${ }^{1,2}$, Lorenzo Crocco ${ }^{2}$, Marta Cavagnaro ${ }^{1,2}$ \\ ${ }^{1}$ CNR-IREA National Research Council of Italy, Institute for Electromagnetic Sensing of Environment, Napoli, Italy, \\ wang.m@irea.cnr.it, crocco.1@irea.cnr.it \\ 2 DIET, Sapienza University of Rome, Rome, Italy, marta.cavagnaro@uniroma1.it
}

\begin{abstract}
Microwave imaging (MWI) for biomedical applications has drawn great attention in recent decades owing to its attractive features such as low cost, portability, and use of non-ionizing radiation. In the microwave imaging system, the antenna plays the most crucial role because it dictates the amount of electromagnetic power transmitted to the human body. In this contribution, an ultra-wideband antipodal Vivaldi antenna was designed for a biomedical MWI system. The antenna is immersed in a coupling medium of permittivity 23 and operates in the frequency band $0.5-5 \mathrm{GHz}$. The radiation of the antenna was improved by removing a piece of substrate from the aperture and replacing it with a high permittivity ceramic cone lens. Such a ceramic lens was capable to increase antenna radiation without changing the antenna impedance. This study paves the way for the assessment of a biomedical microwave imaging system.
\end{abstract}

Index Terms - Vivaldi antenna, Ultra-wideband (UWB), ceramic lens, antenna directivity, microwave imaging

\section{INTRODUCTION}

Microwave imaging (MWI) for biomedical applications has been widely researched in recent decades because it has advantages of low cost, portability, and non-ionizing nature of the exploited radiation, i.e., the electromagnetic field in the microwave (MW) range [1]. In a MWI system, the antenna represents the most important part because it dictates the amount of electromagnetic power transmitted to the human body [2]. Therefore, antenna design for MWI systems has received substantial interest in the last two decades, and a considerable amount of research has been dedicated to this topic [3][4][5].

Several challenges exist when it comes to the design of antennas for MWI systems for biomedical purposes, such as the limitation of availability in the electromagnetic spectrum [6]. So far, most biomedical applications work at 13.55313.567 MHz, 433.1-434.8 MHz, 868-868.6 MHz, 902.8-928 $\mathrm{MHz}, 2.400-2.500 \mathrm{GHz}$, and 5.725-5.875 GHz, which belong to the Industrial, Scientific, and Medical (ISM) band, since unlicensed operations are permitted in these bands [7][8]. Accordingly, most biomedical MWI systems use ultra-wideband (UWB) antennas because they are capable of covering the bandwidth allocated to ISM [8]. A further merit of using wideband antennas is that it could give researchers an additional degree of freedom by selecting a wide range of frequency points to be used in the MWI algorithm [9]. However, the wide bandwidth of the antenna always comes together with the sacrifice of the antenna performances on its directivity and quality factor [2].

Antennas for biomedical applications usually work in close proximity to lossy tissues, where the electromagnetic wave attenuates severely. Therefore, improving the antenna radiation at the higher frequencies is another challenge that researchers have to confront.

Finally, it is known that microwave imaging resolution can be increased by using a greater number of antennas in the system [10]. Thus, it is preferable to use a compact antenna because it could allow placing a greater number of antenna elements in the array configuration [11].

Summarizing the reported considerations, a biomedical MWI system demands an antenna to be compact, wide-band, and with a high directivity to allow good penetration inside the human tissues [9]. In the present paper, an antipodal Vivaldi antenna to be used in a MWI system for the monitoring of thermal ablation was designed. Design requirements, besides those listed above, were a frequency band from $500 \mathrm{MHz}$ to $5 \mathrm{GHz}$, with a possibility to work at a higher frequency, and operation in close proximity of the human abdomen. Several versions of the antenna were then studied to look for improvements in the antenna's radiation without deteriorating antenna's matching or increasing antenna's dimensions.

\section{MATERIALS AND METHODS}

\section{A. Antipodal Vivaldi Antenna (AVA)}

The antipodal Vivaldi antenna (AVA) is an antenna type that has been widely used in MWI systems for biomedical applications thanks to its wide bandwidth, compact dimensions, and end-fire radiation. The antenna proposed in this work was designed following the design steps and equations in [12]. The AVA designed in [12] was placed in the air and operated from 3 to $10 \mathrm{GHz}$. The design study in 


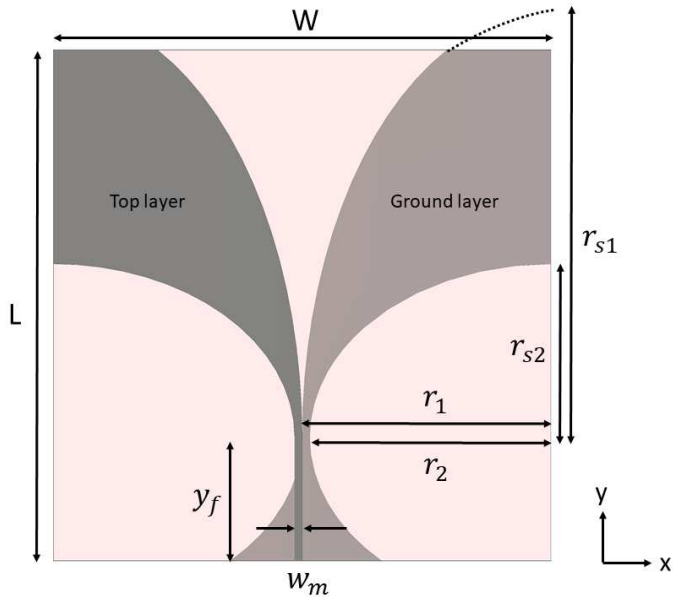

Figure 1 Antipodal Vivaldi Antenna (AVA) geometry (gray: metallic layer, pink: antenna substrate)

this work was focused on taking the desirable features of the AVA antenna, i.e., compactness, end-fire radiation, and wide bandwidth, and translating them into the considered design scenario, in which the antenna radiates in a lower frequency band, i.e. from $500 \mathrm{MHz}$ and onwards. To keep the small antenna's dimensions, the antenna was considered immersed in a coupling medium, as often encountered in MWI systems [13]. The selected medium $\left(\varepsilon_{\mathrm{r}}=23, \sigma=0.07 \mathrm{~S} / \mathrm{m}\right.$, [14]) corresponds to a practical water and oil emulsion that proved to be realizable in [15].

According to the equations ruling the Vivaldi antenna [12], the antenna dimensions are determined by the lowest working frequency. In the present design, to take into account the presence of the coupling medium, the equations of the original design [12] were modified as illustrated here below. The antenna dimension is given by

$$
W=L=\frac{c}{f_{1}} \sqrt{\frac{2}{\varepsilon_{m m}+\varepsilon_{s u b}}}
$$

where $W$ is antenna width, $L$ is antenna length, $c$ is the speed of light in vacuum, $f_{1}$ is the lowest working frequency, $\varepsilon_{m m}$ is the permittivity of the coupling medium, $\varepsilon_{\text {sub }}$ is the permittivity of the substrate.

The input microstrip line has a characteristic impedance given by [16]:

$$
Z_{0}= \begin{cases}\frac{60}{\sqrt{\varepsilon_{e}}} \ln \left(\frac{8 t_{s}}{w_{m}}+\frac{w_{m}}{4 t_{s}}\right) & \text { for } \frac{w_{m}}{t_{s}} \leq 1 \\ \frac{120 \pi}{\sqrt{\varepsilon_{e}}\left[\frac{w_{m}}{t_{s}}+1.393+0.667 \ln \left(\frac{w_{m}}{t_{s}}+1.444\right)\right]} & \text { for } \frac{w_{m}}{t_{s}} \geq 1\end{cases}
$$

where $w_{m}$ is the microstrip line width, $t_{s}$ is the substrate thickness and the effective permittivity $\varepsilon_{e}$ is calculated as:

$$
\varepsilon_{e}=\frac{\varepsilon_{s u b}+\varepsilon_{m m}}{2}+\frac{\varepsilon_{s u b}-\varepsilon_{m m}}{2} \cdot \frac{1}{\sqrt{1+12 t_{s} / w_{m}}}
$$

The radiating part of the antenna is created by the intersection of two-quarters of ellipses, as shown in Figure 1, where the antenna substrate is made transparent in the interest of clarity. The major and minor radii of the large ellipse are indicated as $r_{s l}$ and $r_{l}$, respectively. The major and minor radii of the small ellipse are $r_{s 2}$ and $r_{2}$, respectively. They are defined by the following equations:

$$
\begin{aligned}
& r_{1}=\frac{W+w_{m}}{2} \\
& r_{2}=\frac{W-w_{m}}{2} \\
& r_{s 1}=c \cdot r_{1} \\
& r_{s 2}=b \cdot r_{2}
\end{aligned}
$$

where $\mathrm{b}$ and $\mathrm{c}$ are optimization parameters, which are adjusted to achieve the desired performances, and $w_{m}$ is the microstrip line width. Finally, $y_{f}$ is the microstrip line length.

The antenna was designed on the substrate RT/duriod 6010LM (Rogers Cooperation, USA, $\varepsilon_{\text {sub }}=10.2, t_{s}=1.905$ $\mathrm{mm}$ ), and simulated through CST software (Dassault Systèmes, France). Starting from the numerical values given by eq. (1) - (7) for the chosen specifications, and optimization was performed using CST optimization tool, looking for the antenna's dimensions to achieve the best matching in the frequency band from 0.5 to $5 \mathrm{GHz}$.

\section{B. AVA with Ceramic Lens}

The antipodal Vivaldi antenna (AVA) in section II-A shows the well-known problem that the radiation direction shifts from the broad-side direction at the higher frequencies [18]. This is because the antenna substrate has a lower permittivity value $\left(\varepsilon_{\text {sub }}=10.2\right)$ as compared to the background medium $\left(\varepsilon_{m m}=23\right)$, so that a discontinuity of the wave phase velocity is created when the wave leaves the substrate and enters into the coupling medium. This in turn leads to the distortion of the radiation direction [18].

One simple approach to try to solve this problem is to remove a piece of substrate from the antenna aperture [17], this could effectively avoid the phase velocity discontinuity without complicating the antenna design. In this work, a triangular shape within the antenna's aperture was removed; the base of the triangle was $d_{1}=28 \mathrm{~mm}$ and the height $h_{1}=$ $20.5 \mathrm{~mm}$.

In [18] and [19], a profiled dielectric lens was proved to be able to improve the antenna directivity without changing the antenna impedance. Therefore, to further enhance the antenna directivity, an additional antenna was studied in which the triangular substrate slot was replaced with a piece of ceramic with a higher permittivity as compared to the background medium. This approach could decrease the phase velocity inside the ceramic lens [18]. The ceramic material used for this lens is T-Ceram E-37 (T-Ceram, S.r.o. Czechia, $\varepsilon=37)$. Two types of ceramic lens were suggested for the 


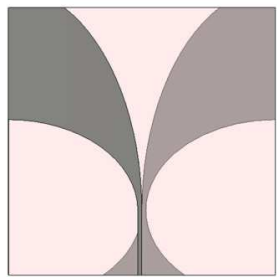

(a)

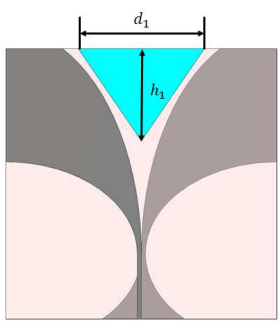

(c)

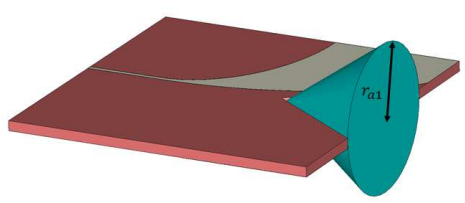

(e)

Figure 3 Antenna geometries (a) Antipodal Vivaldi antenna with the complete substrate (AVA-CS); (b) Antipodal Vivaldi antenna with the removed substrate (AVA-RS); (c) Antipodal Vivaldi antenna with the triangular-shaped lens (AVA-TL); (d) Antipodal Vivaldi antenna with the cone-shaped lens (AVA-CL); (e) 3D view of the AVA-CL. $\boldsymbol{d}_{\mathbf{1}}=28$ $\mathrm{mm}, \boldsymbol{h}_{\mathbf{1}}=20.5 \mathrm{~mm}, \boldsymbol{r}_{\boldsymbol{a} \mathbf{1}}=14 \mathrm{~mm}$ (grey: metallic part; pink: antenna substrate; blue: ceramic lens)

antenna directivity improvement assessment: a triangular shape, whose dimensions fit into the removed antenna substrate, and a cone shape with base radius $r_{a 1}=14 \mathrm{~mm}$, and height $h_{1}=20.5 \mathrm{~mm}$. As compared to the antennas in [18] and [19], the ceramic cone has a symmetrical shape in the xy and the yz-planes which could improve the antenna directivity in both planes. The mentioned four antipodal Vivaldi antennas' geometries are shown in Figure 2, named as antipodal Vivaldi antenna with complete substrate (AVA-CS); antipodal Vivaldi antenna with removed substrate (AVA-RS); antipodal Vivaldi antenna with triangular-shaped lens (AVATL); antipodal Vivaldi antenna with cone-shaped lens (AVACL), separately.

\section{RESUlts AND DiscUSSION}

Table I reports the four antipodal Vivaldi antennas' dimensions achieved through the CST simulations. The simulated $\mathrm{S}_{11}$ parameters are shown in Figure 3. The antennas are capable of working from $0.5-5 \mathrm{GHz}$ inside the coupling medium, with a possibility to work at a higher frequency. Despite the difference in their designs, the matchings of
TABLE I ANTIPODAL VIVALDI ANTENNA DIMENSION AND OPTIMIZATION PARAMETERS

\begin{tabular}{|c|c|}
\hline Parameters & Values (mm) \\
\hline $\mathrm{W}$ & 60 \\
\hline $\mathrm{L}$ & 60 \\
\hline $\mathrm{y}_{\mathrm{f}}$ & 14.5 \\
\hline $\mathrm{w}_{\mathrm{m}}$ & 0.9 \\
\hline $\mathrm{r}_{1}$ & 30 \\
\hline $\mathrm{r}_{2}$ & 29.1 \\
\hline $\mathrm{r}_{\mathrm{s} 1}$ & 50.1 \\
\hline $\mathrm{r}_{\mathrm{s} 2}$ & 20.37 \\
\hline $\mathrm{t}_{\mathrm{s}}$ & 1.905 \\
\hline $\mathrm{b}$ & 0.7 \\
\hline $\mathrm{c}$ & 1.67 \\
\hline
\end{tabular}

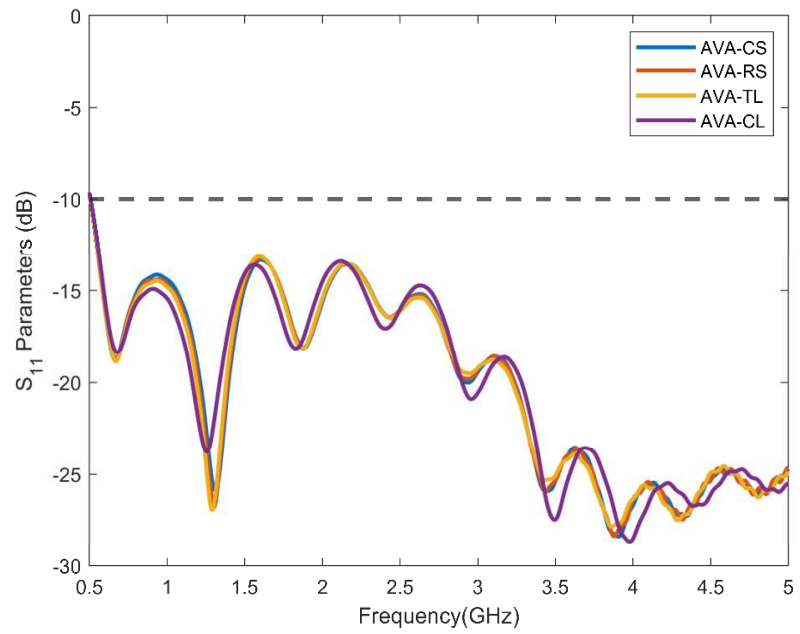

Figure 2 Simulated $S_{11}$ parameters immersed in a matching medium of the four AVA antennas: AVA-CS, AVA-RS, AVA-TL, AVA-CL

antennas are similar. The removal of the antenna substrate and the implementation of extra ceramic materials barely changes the antenna matching. Being the proposed antennas to be used in a microwave imaging system, i.e. in a near field application, the next step would be to verify the matching of the four antennas when located close to the human body. In this respect, it can be noted that the AVA-CS antenna already proved to keep the matching when located close to a simple model of the human abdomen [14].

The E-field distributions of the four antennas are shown in Figure 4 (panel a plane xy, panel b plane yz). It is obvious that the four AVA share similar radiation performances below $2 \mathrm{GHz}$. However, the direction of maximum radiation of AVA-CS is shifting from the boresight as the frequency increases above $3 \mathrm{GHz}$, with a reduction of the radiated field on the xy-plane (Figure 4 (a)), especially at $5 \mathrm{GHz}$. The AVA-RS shows an enhancement in the electric field at higher frequencies after removing the substrate. However, asymmetry in the radiated field in the yz-plane (Figure 4 (b)) is still observed above $4 \mathrm{GHz}$. The E-field of the AVA-TL shows improved radiation mainly in the yz-plane as 
compared to the antenna without a lens. The AVA-CL shows improved radiation above $2 \mathrm{GHz}$ in both planes as compared to the other three antennas. Owing to the symmetrical shape of the cone lens, the AVA-CL shows symmetrical radiation in the xy and the yz-planes. three antennas. The ceramic lens proved capable of enhancing the antenna radiation without changing the antenna matching. Future work could include simulation of the antenna with the presence of a human phantom, the realization of the antipodal Vivaldi antennas with the coneshaped lens, and the measurement of the $S_{11}$ parameter when
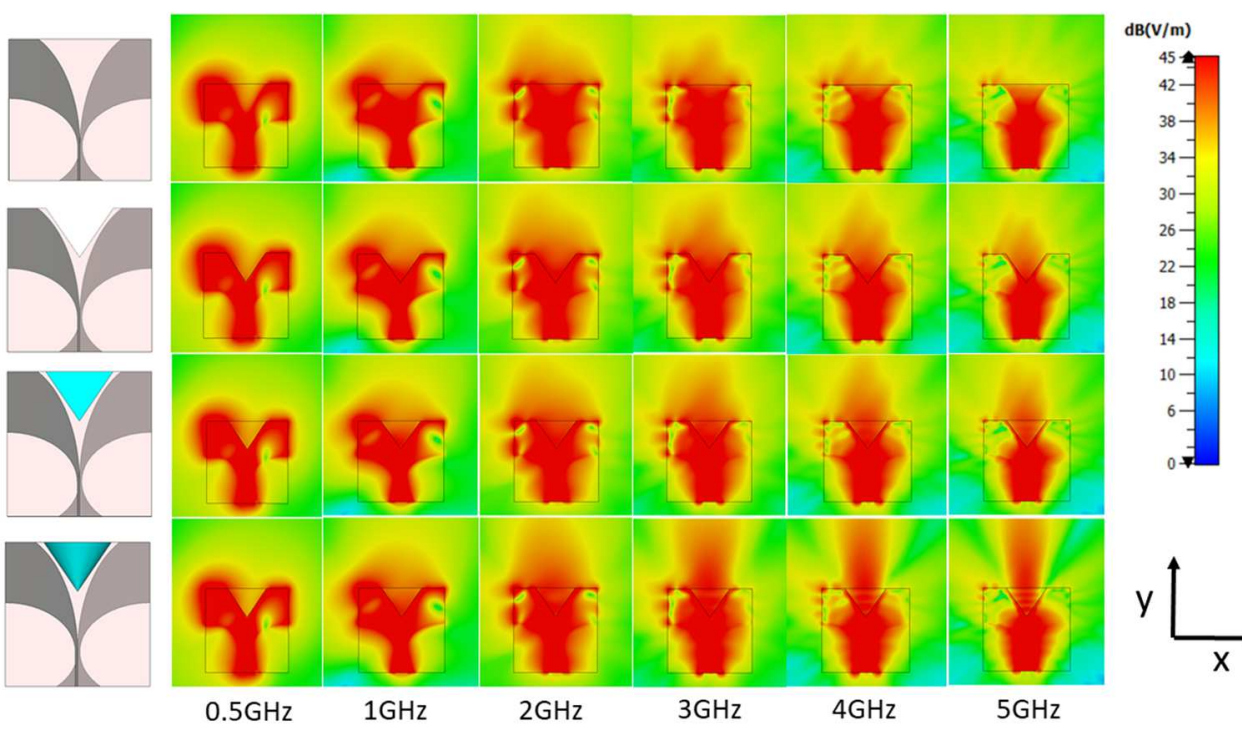

$1 \mathrm{GHz}$

$2 \mathrm{GHz}$

$3 \mathrm{GHz}$

$4 \mathrm{GHz}$

$5 \mathrm{GHz}$

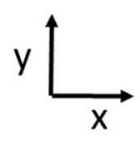

(a)
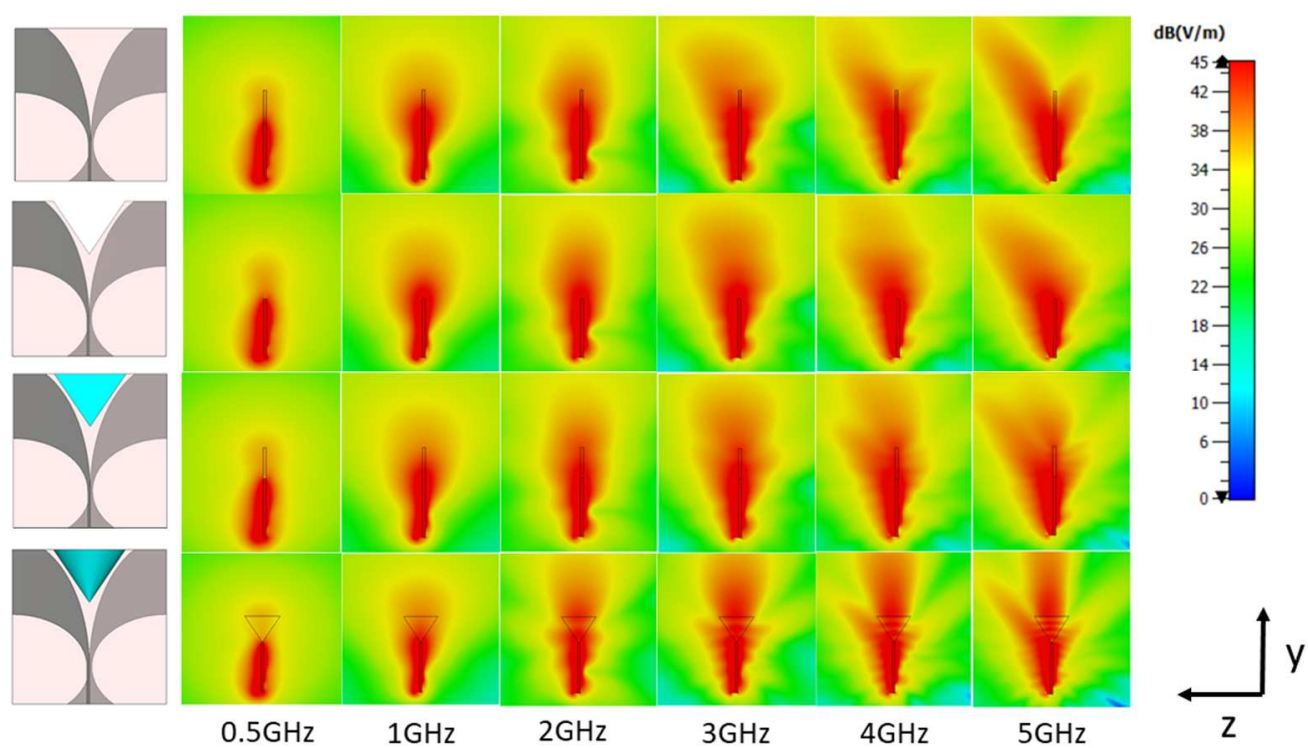

(b)

Figure 4 E-field distribution of the four Vivaldi antennas: (a) at xy-plane; (b) at yz-plane. From the top to the bottom are: AVA-CS, AVA-RS, AVA-TL, and AVA-CL. The antennas are immersed in a matching medium $\left(\varepsilon_{\mathrm{r}}=23, \sigma=0.07 \mathrm{~S} / \mathrm{m}\right)$

\section{CONCLUSIONS}

In this work, four antipodal Vivaldi antennas for biomedical microwave imaging systems were designed and numerically assessed. Among these, the antipodal Vivaldi antenna with a cone-shaped lens (AVA-CL) shows the highest radiation from 2 to $5 \mathrm{GHz}$ as compared to the other placing it inside the coupling medium.

\section{ACKNOWLEDGMENT}

This work was supported by the EMERALD project funded from the European Union's Horizon 2020 research and 
innovation program under the Marie Skłodowska-Curie grant agreement No. 764479.

\section{REFERENCES}

[1] M. Pastorino, "Microwave imaging," Vol. 208, John Wiley \& Sons, 2010.

[2] B. Borja, J. A. Tirado, \& H. Jardon, "An Overview of UWB Antennas for Microwave Imaging Systems for Cancer Detection Purposes," Progress In Electromagnetics Research B, 80, 2018.

[3] B. A. J. Mohammed, A. M. Abbosh, \& P. Sharpe, "Planar array of corrugated tapered slot antennas for ultrawideband biomedical microwave imaging system," International Journal of RF and Microwave Computer-Aided Engineering, 23(1), 59-66, 2013.

[4] G. Adamiuk, T. Zwick, \& W. Wiesbeck, "Compact, dual-polarized UWB-antenna, embedded in a dielectric," IEEE Transactions on Antennas and Propagation, 58(2), 279-286, 2009.

[5] X. Li, M. Jalilvand, Y. L. Sit, \& T. Zwick, "A compact double-layer on-body matched bowtie antenna for medical diagnosis," IEEE transactions on antennas and propagation, 62(4), 1808-1816, 2014.

[6] A. Yakovlev, S. Kim, \& A. Poon, "Implantable biomedical devices: Wireless powering and communication," IEEE Communications Magazine, 50(4), 152-159, 2012.

[7] J. C. Bolomey, \& L. Jofre, "Three decades of active microwave imaging achievements, difficulties and future challenges," In 2010 IEEE International Conference on Wireless Information Technology and Systems (pp. 1-4). IEEE, 2010, September.

[8] A. Kiourti, \& K. S. Nikita, "A review of implantable patch antennas for biomedical telemetry: Challenges and solutions" [wireless corner]. IEEE Antennas and Propagation Magazine, 54(3), 210-228, 2012.

[9] X. Li, E. J. Bond, B. D. Van Veen, \& S. C. Hagness, "An overview of ultra-wideband microwave imaging via space-time beamforming for early-stage breast-cancer detection", IEEE Antennas and Propagation Magazine, 47(1), 19-34, 2005.

[10] O. M. Bucci, L. Crocco, R. Scapaticci, \& G. Bellizzi, "On the design of phased arrays for medical applications", Proceedings of the IEEE, 104(3), 633-648, 2016.

[11] T. Sugitani, S. Kubota, A. Toya, X. Xiao, \& T. Kikkawa, "A Compact 4 by 4 Planar UWB Antenna Array for 3-D Breast Cancer Detection," IEEE Antennas and Wireless Propagation Letters, 12, 733-736, 2013.

[12] A. M. Abbosh., H. K. Kan., \& M. E. Bialkowski, "Compact ultrawideband planar tapered slot antenna for use in a microwave imaging system," Microwave and optical technology letters, 48(11), 2212-2216, 2006.

[13] N. K. Nikolova, "Microwave imaging for breast cancer," IEEE microwave magazine, 12(7), 78-94, 2011.

[14] M. Wang, L. Crocco, M. Cavagnaro, "On the Design of a Microwave Imaging System to Monitor Thermal Ablation of Liver Tumors", IEEE Journal of Electromagnetics, RF and Microwaves in Medicine and Biology, 2021.

[15] J. Stang, M. Haynes, P. Carson, and M. Moghaddam, "A preclinical system prototype for focused microwave thermal therapy of the breast," IEEE Transactions on Biomedical Engineering, vol. 59, no. 9, pp. $2431-2438,2012$.

[16] D. M. Pozar, "Microwave engineering," John wiley \& sons, 2009.

[17] N. N. Wang, M. Fang, H. T. Chou, J. R. Qi, \& L. Y. Xiao, "Balanced antipodal Vivaldi antenna with asymmetric substrate cutout and dualscale slotted edges for ultrawideband operation at millimeter-wave frequencies," IEEE Transactions on Antennas and Propagation, 66(7), 3724-3729, 2018.

[18] J. Bourqui, M. Okoniewski, \& E. C. Fear, "Balanced antipodal Vivaldi antenna with dielectric director for near-field microwave imaging," IEEE Transactions on Antennas and Propagation, 58(7), 2318-2326, 2010.

[19] R. Cicchetti, A. Faraone, E. Miozzi, R. Ravanelli \& O. Testa, "A highgain mushroom-shaped dielectric resonator antenna for wideband wireless applications," IEEE Transactions on Antennas and Propagation, 64(7), 2848-2861, 2016. 